\begin{tabular}{|c|c|}
\hline Title & Constant Margin-A ngle Control of Commutatorless Motor Using Microcomputer \\
\hline Author(s) & Fukuda, Shoji; Sasaka, Shinji \\
\hline Citation & IEEE Transactions on Industry A pplications, 23(5), 855-862 \\
\hline Issue Date & 1987-09 \\
\hline Doc URL & http:/hdl .handle.net/2115/5965 \\
\hline Rights & $\begin{array}{l}\text { O1987 IEEE. Personal use of this material is permitted. However, permission to reprint/republish this material for } \\
\text { advertising or promotional purposes or for creating new collective works for resale or redistribution to servers or lists, } \\
\text { or to reuse any copyrighted component of this work in other works must be obtained from the IEEE." } \\
\text { IEEE, IEEE Transactions on Industry A pplications } \\
\text {, 23(5), 1987, p855-862 }\end{array}$ \\
\hline Type & article \\
\hline File Information & ITIA 23_5.pdf \\
\hline
\end{tabular}

Instructions for use 


\title{
Constant Margin-Angle Control of Commutatorless Motor Using Microcomputer
}

\author{
SHOJI FUKUDA, MEMBER, IEEE, AND SHINJI SASAKA
}

\begin{abstract}
Constant margin-angle control is a triggering scheme that can eliminate the commutation failure and increase the power factor and efficiency of commutatorless motors. The described scheme does not need to detect the margin angle, but the dc current and overlap angle are detected instead. The margin angle is controlled by using a correction table of the lead angle in terms of the dc current, which provides a practical solution to implement the constant margin-angle control. The problems introduced by the scheme, the occurrence of instability and commutation failure, are discussed, and methods to eliminate them are presented. Experimental results confirm the validity of this scheme.
\end{abstract}

\section{INTRODUCTION}

$\mathrm{T}_{\mathrm{f}}^{\mathrm{H}}$ HE commutatorless motor (CLM) is a synchronous motor fed by a current source inverter, where the constant margin-angle control scheme has been proposed for the improvement of the motor performances, i.e., elimination of commutation failure and increase in power factor and efficiency [1], [2].

There are two ways to implement constant margin-angle control.

1) The margin angle $\delta$ is detected and is directly controlled [3].

2) The dc link current $I d$ is detected and $\delta$ is indirectly controlled by using a function generator [1], [2] or a correction table [4] in terms of Id.

The first way seems to be better because $\delta$ can be kept constant in a transient state as well as a steady state. However, detecting $\delta$ over a wide speed range is difficult, since ripple voltages generated by a rectifier appear in the terminal voltages of CLM and disturb to detect the zero crossing point of the terminal voltages [3]. On the other hand, the second way is considered to be more practical and applicable to adjustable CLM drives because the detection of $\delta$ is not needed. References concerning the second way dealt with a steadystate performance, but a transient performance is also important to put the scheme to a practical use.

In this paper the second way, mainly under a transient state, is treated, in which the overlap angle $u$ is detected and used to improve the transient performance of CLM. The entire speed

Paper IPCSD 86-67, approved by the Industrial Drives Committee of the IEEE Industry Applications Society for presentation at the 1986 Industry Applications Society Annual Meeting, Denver, CO, September 28-October 3. Manuscript released for publication January 22, 1987.

S. Fukuda is with the Department of Electrical Engineering, Hokkaido University, North-13, West-8, Kita-ku, Sapporo, 060 Japan.

S. Sasaka is with the Nippon Steel Corporation, Mororan Works, Midorigaoka-Dai-2-Ryou, 1-2-21, Muroran, 050 Japan.

IEEE Log Number 8714613. regulator system is illustrated in Fig. 1. Constant- $\delta$ control is demonstrated by the feedback loop using Table I. The relationship between the dc current and the lead angle has been calculated in advance under the assumption of the steady-state operation with $\delta=20^{\circ}$, and has been tabulated in the ROM of a microcomputer. The microcomputer looks up the table and corrects the lead angle $\beta_{0}$ in terms of the dc current $I d$ and the overlap angle $u$.

The constant $-\delta$ control scheme using the table raises two problems that must be elucidated and solved to put the scheme to a practical use. The first problem is the problem concerning instability.

The constant- $\delta$ control scheme has intrinsic instability. This is due to the voltage drop in induced voltage during the commutation period [5]. In this paper, the Routh-Hurwitz criterion is applied to the linearized CLM equations with constant $-\delta$ control, and stability investigations are made at every operating point in the current-versus-speed plane. The results show that CLM becomes unstable in heavy-load and/or high-speed operation. A method to stabilize the CLM system is presented.

The second problem concerns the transient behavior of the margin angle. Since Table I has been determined under the steady-state condition, $\delta$ in a transient state does not always coincide with the reference values of $20^{\circ}$, which may cause commutation failure if $\delta$ reduces remarkably. The variations in $\delta$ in a transient state are calculated by a digital simulation technique. The results indicate that the commutation failure will occur when a rapid increase in $I d$ occurs, if there is a relatively long delay time in the gate pulse generators of an inverter. A method to prevent the commutation failure is also presented.

Finally the constant- $\delta$ controlled CLM speed regulator is implemented by use of a microcomputer. Stable operation with the high power factor is confirmed by means of the experimental results.

\section{Stability InVESTigation}

\section{A. Instability in the Constant- $\delta$ Control}

The salient-pole CLM can be represented by the following nonlinear differential equations [6]:

$$
\begin{gathered}
\Psi d o=-(3 \sqrt{3} / \pi) I d \cdot L a d d \sin \left(\beta_{0}-u / 2\right)+L d d o \cdot i d d \\
\Psi d d=\Psi d o+L f d d \cdot I f
\end{gathered}
$$

$$
\Psi q o=(3 \sqrt{3} / \pi) I d \cdot L a d q \cos \left(\beta_{0}-u / 2\right)+L d q o \cdot i d q
$$




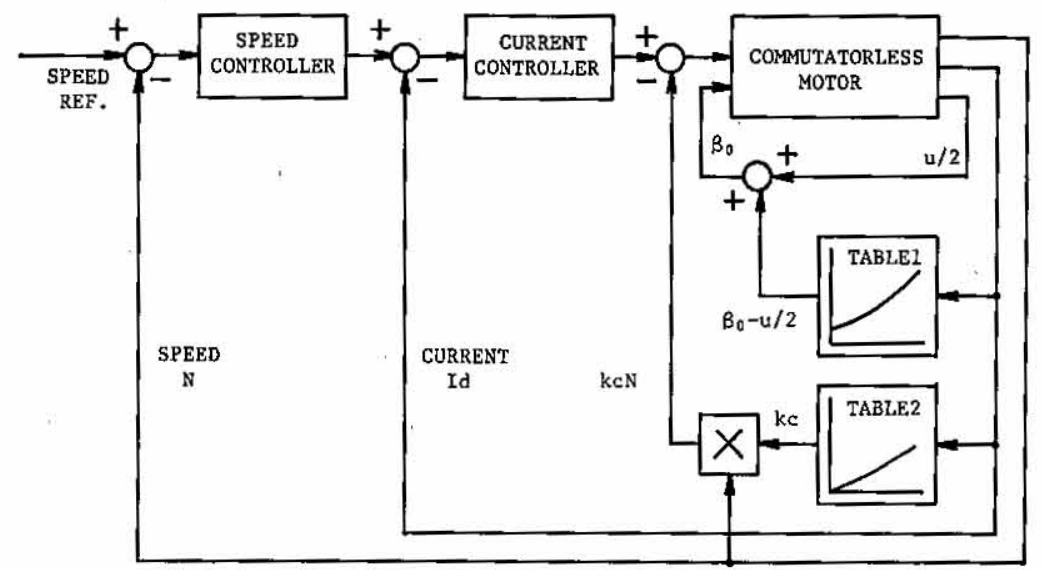

Fig. 1. Control block diagram of commutatorless motor with constant margin-angle control using ROM table.

TABLE I

CONSTANTS OF THE MOTOR

\begin{tabular}{lc}
\hline Power & $1 \mathrm{~kW}$ \\
Pole number & 4 \\
Rated voltage & $100 \mathrm{~V}$ \\
$R$ & $3.171 \Omega$ \\
$L d c$ & $95 \mathrm{mH}$ \\
$L d d o$ & $27.6 \mathrm{mH}$ \\
$L d q o$ & $17.1 \mathrm{mH}$ \\
$L s^{\prime \prime}$ & $4.74 \mathrm{mH}$ \\
$r f$ & $19 \Omega$ \\
$L f$ & $7.76 \mathrm{H}$ \\
$r d d$ & $2.55 \Omega$ \\
$r d q$ & $2.65 \Omega$ \\
$L a f$ & $316 \mathrm{mH}$ \\
$L a d d$ & $20 \mathrm{mH}$ \\
$L a d q$ & $11.2 \mathrm{mH}$ \\
$L f d d$ & $413 \mathrm{mH}$ \\
$J$ & $0.33 \mathrm{~N} \cdot \mathrm{m} \cdot \mathrm{s}^{2}$ \\
$R \omega$ & $0.0161 \mathrm{~N} \cdot \mathrm{m} \cdot \mathrm{s}$ \\
\hline
\end{tabular}

$$
\begin{gathered}
P \cdot \Psi d d+r d d \cdot i d d=0 \\
P \cdot \Psi q o+r d q \cdot i d q=0 \\
V f=(L a f / L a d d) P \cdot \Psi d d+(l f \cdot P+r f) I f \\
A=p \cdot \omega m(L a f \cdot I f+k d \cdot \Psi d o)+P \cdot k q \cdot \Psi q o \\
B=-p \cdot \omega m \cdot k q \cdot \Psi q o+P(L a f \cdot I f+k d \cdot \Psi d o) \\
A n=p \cdot \omega m(L a f \cdot I f+k d \cdot \Psi d o), \\
\quad B n=-p \cdot \omega m \cdot k q \cdot \Psi q o \\
\sqrt{2} V i=\sqrt{A^{2}+B^{2}}, \tan (\alpha)=-B / A, k d=L a d d / L d d o \\
k q=L a d q / L d q o, E x=3 p \cdot \omega m \cdot L s^{\prime \prime} \cdot I d / \pi \\
=3 \sqrt{6} V i \cos \left(\beta_{0}-\alpha-u / 2\right)+\cos \left(\beta_{0}-\alpha\right) /(2 \pi) \\
E d=3 \sqrt{6} V i \cos \left(\beta_{0}-\alpha\right) / \pi+E x \\
V d=(L d c \cdot P+R) I d+E d
\end{gathered}
$$

$$
\psi g=\sqrt{A^{2}+B^{2}} /(p \cdot \omega m), \eta=\cos \left(\beta_{0}-\alpha-u / 2\right),
$$

$$
\begin{array}{r}
T a v=(3 \sqrt{3} / \pi) p \cdot I d \cdot \psi g \cdot \eta \\
T a v=(J \cdot P+R \omega) \omega m+T L
\end{array}
$$

where $\xi \equiv \cos \left(\beta_{0}-u / 2\right) ; \Psi d d, \Psi q o$ denote the total magnetic flux linkages of the $d$-axis and $q$-axis damper windings, respectively; and $I f, \omega m$ denote the field current and angular speed of the motor, respectively. To elucidate the cause of the instability in the constant $\delta$ control scheme, let us consider a damperless cylindrical-pole CLM as a simple example. The dc counter EMF is obtained by

$$
E d=(3 \sqrt{3} / \pi) p \cdot \omega m \cdot L a f \cdot I f \cdot \xi .
$$

In the constant- $\delta$ control scheme, the lead angle $\beta_{0}$ is increased with an increase in dc current Id. That is,

$$
\Delta \xi / \Delta I d \equiv-K \xi<0 .
$$

This means that the dc counter EMF is decreased as the dc current increases, which is an intrinsic characteristic in the constant- $\delta$ control. If $K \xi$, is large enough, the unstable behavior of CLM may occur. The saliency of the motor furthers this characteristic. The contents of. Table I used in the constant- $\delta$ control loop are illustrated in Fig. 2. The parameters of the tested CLM are given in Table I.

Equations (1)-(5) are linearized by assuming small perturbations in the vicinity of equilibrium points, then $\Delta \xi$ is replaced by $-K \xi \cdot \Delta I d$, which makes up the linearized CLM equations with constant- $\delta$ control [7]. The equilibrium point is determined by $\left(\beta_{0}-u / 2\right) o$, Ifo, Ido, and $\omega m o$, but $\left(\beta_{0}-u\right.$ / 2) $o$ and Ido must be chosen out of the values on the $\left(\beta_{0}-u\right.$ / 2)-versus-Id curve in Fig. 2.

Stability discrimination is carried out by the application of the Routh-Hurwitz criterion to the linearized equations. The results are illustrated in Fig. 3, where the region below the curve is stable and above is unstable. The figure indicates that CLM becomes unstable if $K \xi$ is large, and gets unstable at a smaller value of $K \xi$ as the speed becomes high. $K \xi$ increases 


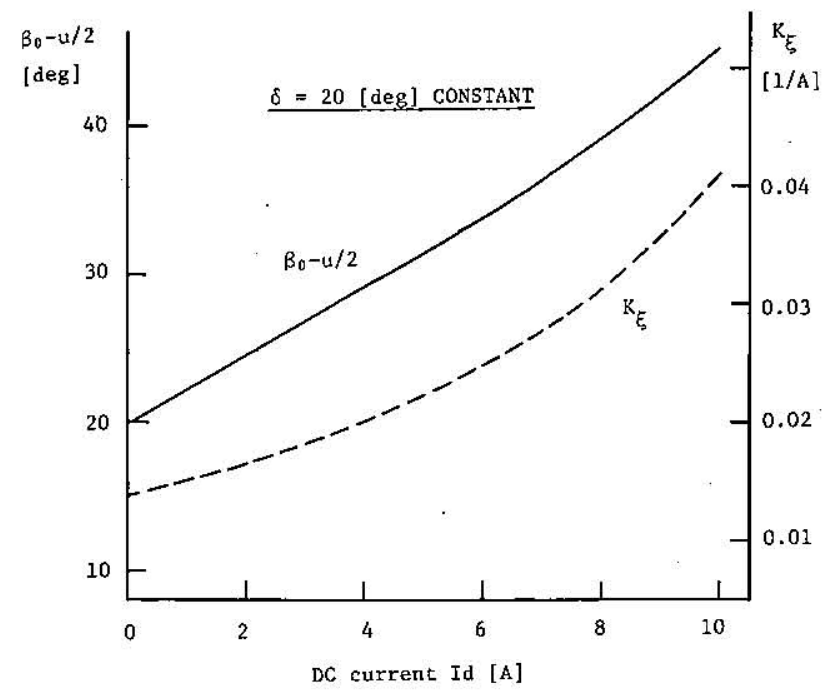

Fig. 2. Characteristics of $\beta_{0}-u / 2$ and $K \xi$ versus de current under margin angle $=20^{\circ}$ constant.

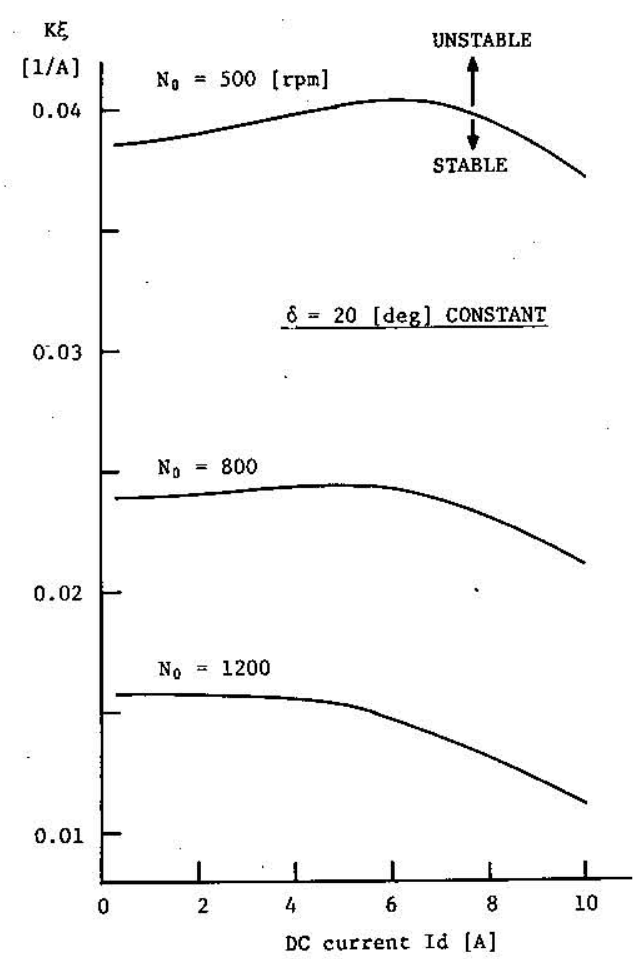

Fig. 3. Stability limits under constant margin-angle control.

with an increase in the dc current, as depicted by the broken line in Fig. 2, therefore a stable region in the current-versusspeed plane can be obtained as Fig. 4 . It should be noted that the stability depends on the speed and dc current (therefore, load). As the speed becomes high and/or load becomes heavy, CLM is liable to be unstable.

\section{B. Stabilization by Means of the EMF Compensation}

As described above, the instability of the constant- $\delta$ controlled CLM is caused by a decrease in the dc counter EMF with an increase in the dc current. The compensation of the decrease in the EMF can stabilize the system. The way of the

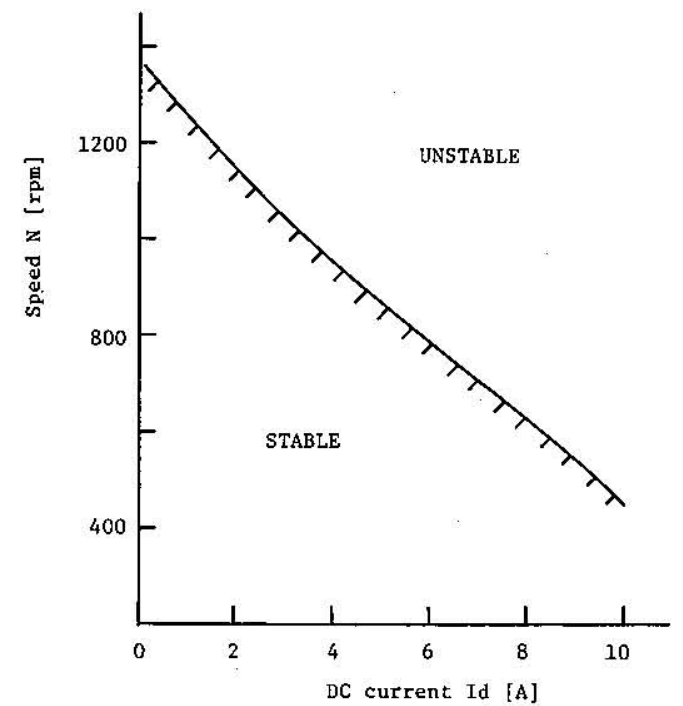

Fig. 4. Stable operation region under constant margin-angle control.

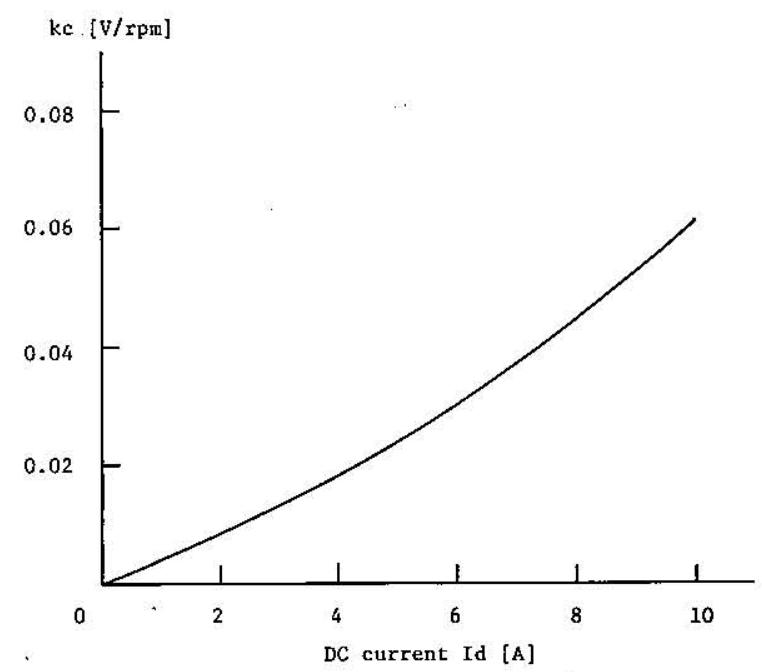

Fig. 5. Feedback gain in dc counter EMP compensation loop.

compensation is demonstrated by the feedback loop using Table $\Pi$ in Fig. 1. The loop gain $k c$ is determined by

$$
k c=(E d o-E d) / N
$$

and is illustrated in Fig. 5, where $E d$ and $E d o$ are the dc counter EMF under the steady-state conditions at load and at no-load, respectively.

Since the gain depends nonlinearly on the dc current, it has been tabulated as in Table II. Fig. 6 shows the loci of the principal roots of the linearized constant- $\delta$ controlled CLM characteristic equations with the EMF compensation. No root exists in the right half-plane, which indicates that the system is stabilized. Fig. 7 shows the step responses to $\Delta V d=8 \mathrm{~V}$. The initial operating point is $N o=485 \mathrm{r} / \mathrm{min}, I d o=2 \mathrm{~A}$, which denotes a stable operating point. Fig. 7(a) is without compensation and Fig. 7(b) with compensation. In Fig. 7(a) the transient attenuates slowly with oscillations, which suggests that the system is underdamped, whereas in Fig. 7(b) it decays quickly. Accordingly, it can be seen that the compensa- 


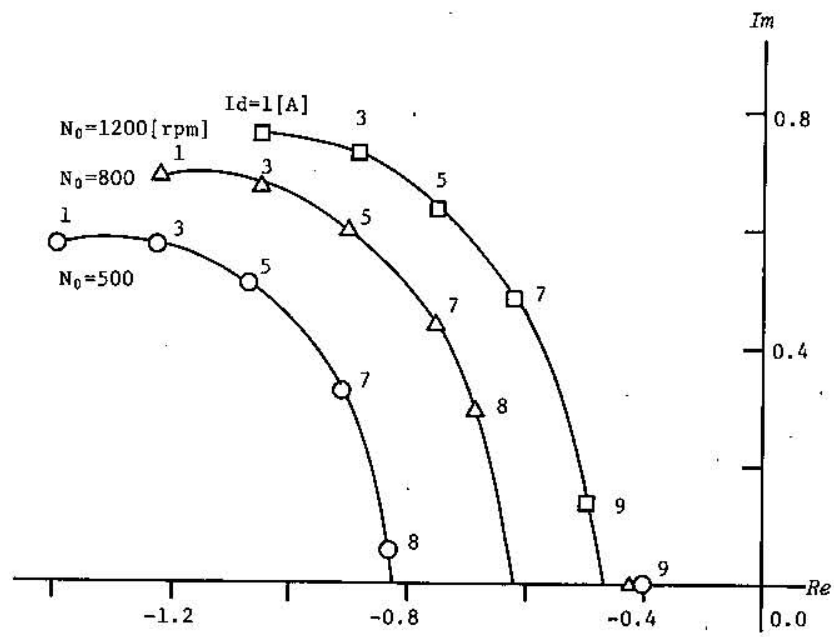

Fig. 6. Loci of principal roots of constant margin-angle controlled CLM with counter EMF compensation.
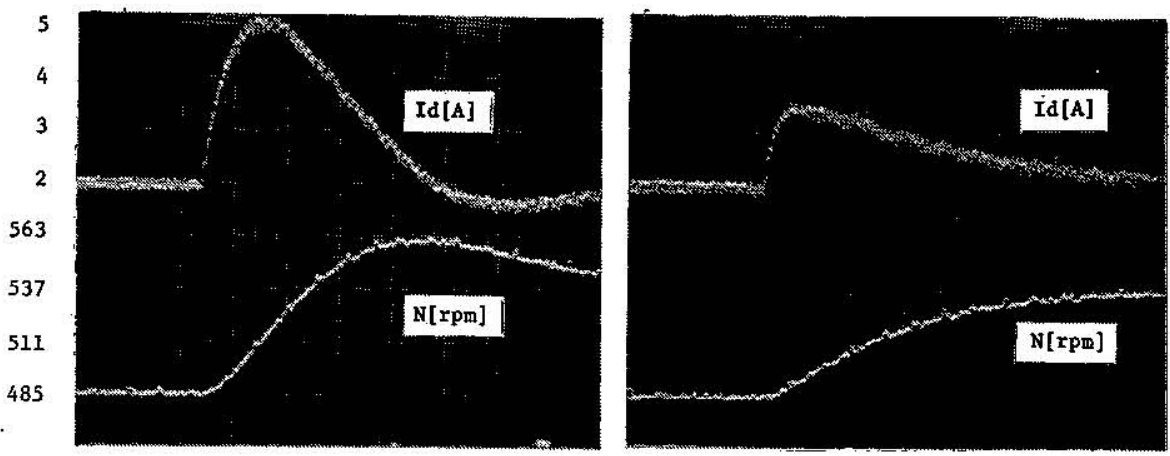

Fig. 7. Step responses of dc current and speed to $\Delta V=8 \mathrm{~V}$. Horizontal; $0.2 \mathrm{~s} / \mathrm{div}$. (a) Without EMF compensation. (b) With EMF compensation.

tion is effective to stabilize the constant- $\delta$ controlled CLM system behavior.

\section{Investigation of Transient Behavior of Margin Angle}

\section{A. Definition of the Transient under Consideration}

The constant $\delta$ control scheme using a table has an inherent problem with commutation. Since the table is determined under a steady-state condition, there is no guarantee if $\delta$ is kept at an expected reference value under a transient condition. Thus to investigate the behavior of $\delta$ in a transient state is essential for realizing adjustable CLM drives with this scheme.

Most of adjustable CLM drive systems include a currentcontrol loop inside a speed-control loop, as shown in the experimental system of Fig. 1. In these cases the dc current is controlled by the current-control loop so as to follow a current reference quickly given by the speed-control loop. If this is the case, (1)-(5) can be approximated by a current-input model [8] instead of a voltage-input model:

$P \cdot \Psi d d=\left\{-(3 \sqrt{3} / \pi) L a d d \cdot I d \sin \left(\beta_{0}-u / 2\right)-\Psi d d\right.$

$$
+L f d d \cdot I f\}(r d d / L d d o)
$$

$$
\begin{gathered}
\begin{aligned}
P \cdot \Psi d q=\left\{(3 \sqrt{3} / \pi) L a d q \cdot I d \cos \left(\beta_{0}-u / 2\right)\right. \\
-\Psi d q\}(r d q / L d q o) \quad(10)
\end{aligned} \\
P \cdot I f=\left\{-\left(r f+\frac{L a f \cdot L f d d}{L a d d \cdot L d d o} r d d\right) I f\right. \\
+\frac{L a f \cdot L f d d}{L d d o}(3 \sqrt{3} / \pi) I d \cdot \sin \left(\beta_{0}-u / 2\right)
\end{gathered}
$$


In such systems the largest transient occurs when a large step change is imposed on the speed reference, because it requires a large step increase or decrease in the current reference. The current responses to that step change may be approximated exponentially: for increasing $I d$

$$
I d=I m+(I o-I m) \exp (-t / \tau)
$$

for decreasing $I d$

$$
I d=I o+(I m-I o) \exp (-t / \tau)
$$

where $I o$ and $I m$ denote a no-load and maximum permissible current, respectively, and $\tau$ denotes the time constant of a step current response. It is noted that these two equations take account of the largest transient possible.

\section{B. Transient Behavior of the Margin Angle}

Digital simulation is carried out to the current-input model. The results are illustrated in Fig. 8, where the solid lines show the responses of $\delta$ when $I d$ is increased as represented in (13). $\delta$ exceeds the reference value $20^{\circ}$, then decreases gradually to $20^{\circ}$. Therefore, no problem is considered to exist with the commutation.

The broken lines show the responses to a decrease in $I d$ as expressed in (14). $\delta$ decreases slightly below $20^{\circ}$ at a time rate determined by the speed of the current-control loop, then rises gradually toward $20^{\circ}$. But there seems to be no problem if $20^{\circ}$ is taken into account as a margin, because the amount of the decrease is only several degrees.

In the experimental setup, however, there was a delay time for feeding gate pulses to inverter thyristors. It was due to the use of counters for the gate pulse generators. The delay was the time interval after a value of $\beta_{0}$ was put on the counter and before the gate pulse was fed to a corresponding thyristor. The delay time is represented by

$$
T d=\left(\pi / 2-\beta_{0}\right) /(p \cdot \omega m)
$$

where $p$ is the number of pole pairs and $T d$ is nearly one sampling period of the margin-angle control routine. Since this delay is inversely proportional to the rotating speed of CLM, it has a serious effect on the transient behavior of $\delta$, especially in low-speed operation.

The simulation results are illustrated in Fig. 9. They indicate that $\delta$ has a sharp dent from its reference value when $I d$ is increased. The dent becomes deeper as the speed is slower, and also becomes deep if the response of the currentcontrol loop is fast, which will cause failure in the commutation at the first commutation after $I d$ is increased. In this situation in the experimental system, the constant $\delta$ control is switched to the constant lead-angle control with $\left(\beta_{0}-u / 2\right)=$ $50^{\circ}$, and increasing $I d$ is delayed by one sampling period when $I d$ must be increased in accordance with a step rise in the speed reference. In this way the commutation failure can be avoided.

When $I d$ is decreased as in (14), on the contrary the simulation results (not shown) indicate that $\delta$ shows a sharp rise beyond the reference value, then reduces toward $20^{\circ}$.

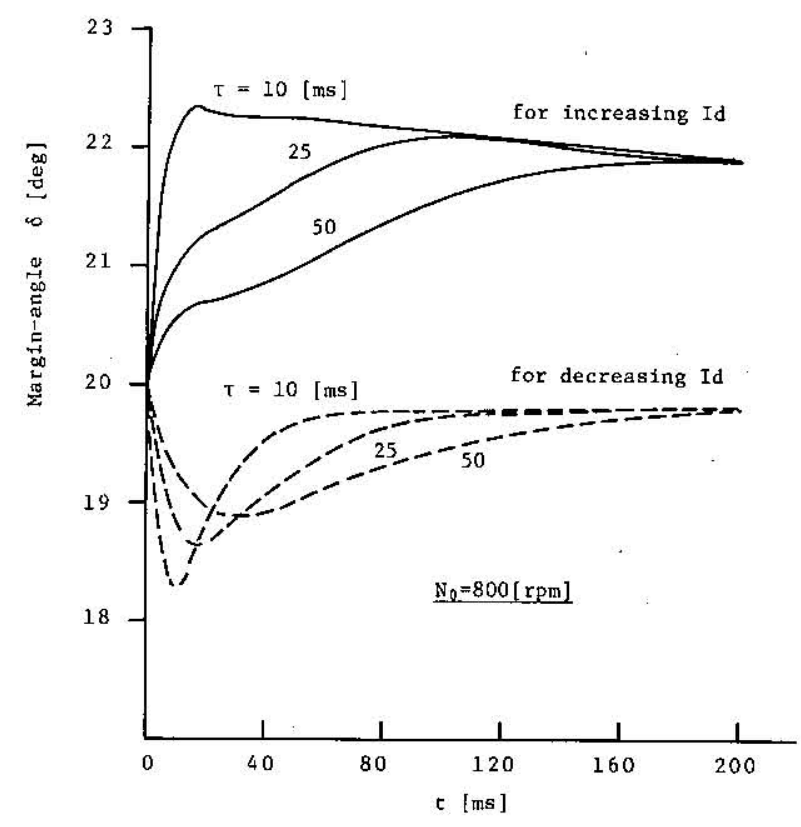

Fig. 8. Transient behavior of margin angle.

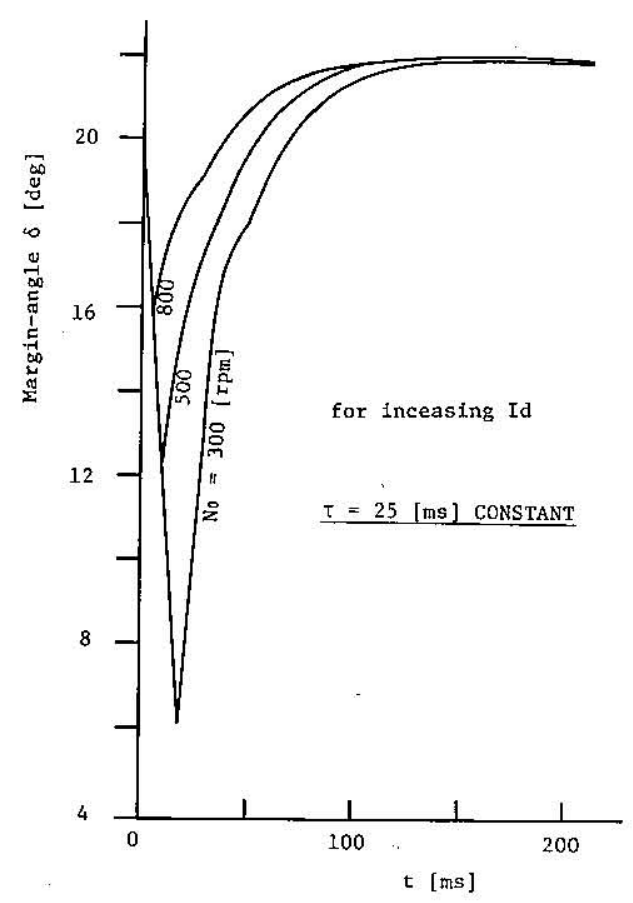

Fig. 9. Transient behavior of margin angle, taking account of delay time of gate pulse generators.

Consequently, excepting the dent, the variations in $\delta$ are all on the safety side in terms of the commutation.

\section{EXPERIMENTAL SYSTEM}

The configuration of the tested setup is illustrated in Fig. 10, and the control block diagram is in Fig. 1. There are three major control processing routines, all of which run on an interrupt basis.

The speed-control processing routine is illustrated in Fig. 11. At first, speed error is calculated. When the error is smaller than a specified value, i.e., $-n_{1}$, a regenerative braking mode is chosen as a control mode. In this mode the $\mathrm{dc}$ 


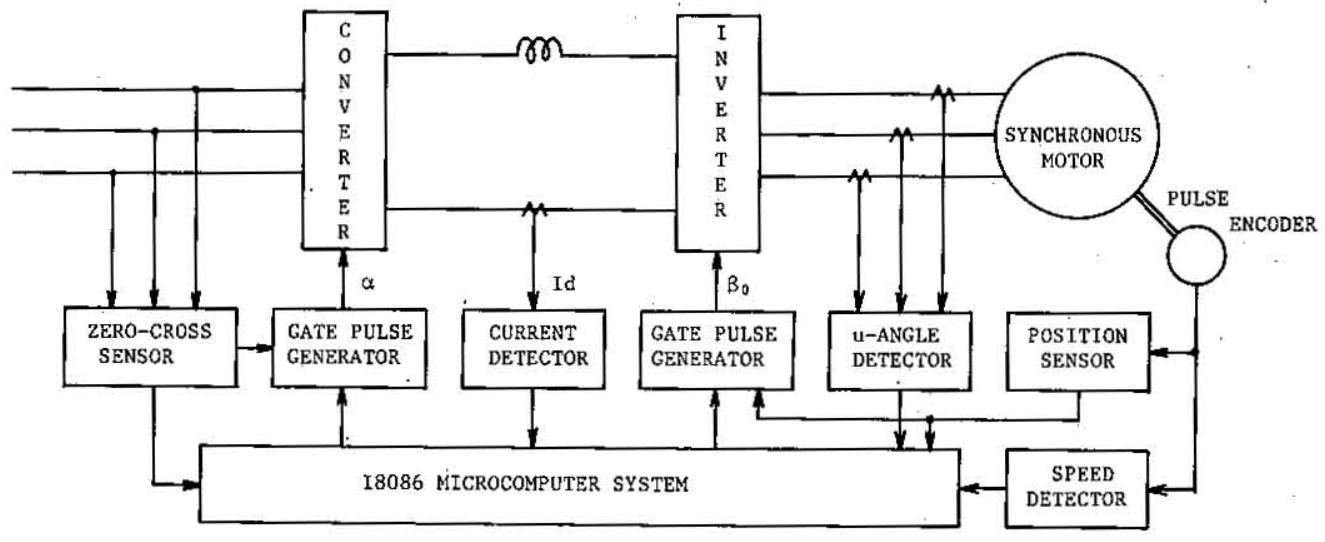

Fig. 10. Configuration of experimental system.

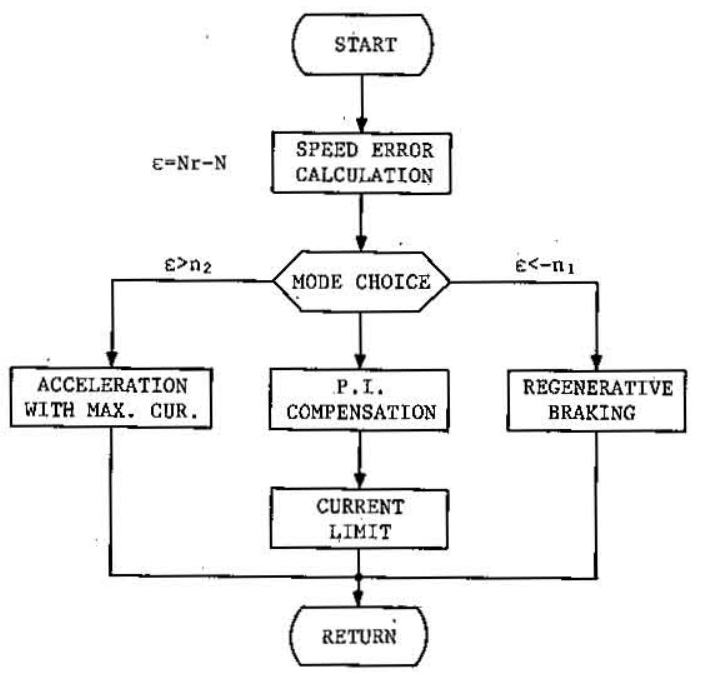

Fig. 11. Flow chart of speed control.

current is reduced to zero, then the motor side converter starts rectifier operation and the line side converter does inverter operation. Accordingly, the direction of the dc voltage is reversed and regenerative braking is performed. When the error is larger than $n_{2}$, an acceleration with a maximum current mode is selected. In this mode the output of a current reference is suspended, and the margin-angle control routine undertakes its output instead. If this is the transition time to the acceleration mode from the other control mode, a transition index is set for processing to prevent the commutation failure.

In the case when the error is $-n_{1}<\epsilon<n_{2}$, the usual proportional/integral (PI) compensation is executed, and a current reference is given to the current controller: This routine runs every $8.33 \mathrm{~ms}$.

In the current-control processing routine, the dc counter EMF compensation, PI compensation, and $\cos ^{-1}$ compensation are carried out. Finally the firing angle is fed to the gate pulse generators of the rectifier. This routine runs every 3.33 ms.

The margin-angle control processing routine is illustrated in Fig. 12. At first, whether or not the present mode is the acceleration mode is checked. If it is, then whether or not the transition index has been set is checked. If the index has been set, the lead angle $\left(\beta_{0}-u / 2\right)$ is determined as $50^{\circ}$ constant

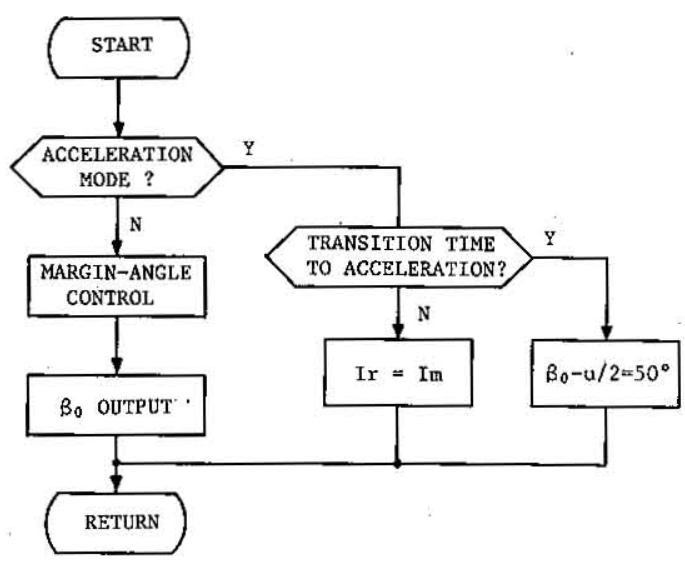

Fig. 12. Flow chart of margin-angle control.

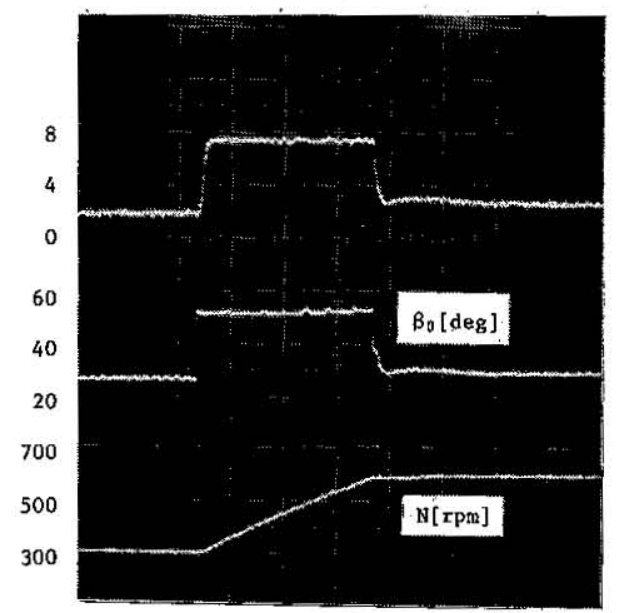

Fig. 13. Responses of dc current, lead angle, and speed to step change in speed reference, $300-600 \mathrm{r} / \mathrm{min}$. Horizontal; $0.5 \mathrm{~s} /$ div.

and the output of the current reference, the maximum value $I m$, is delayed by one sampling period during the acceleration. This processing means that the current is raised after the gate pulse of $50^{\circ}$ is sent to the inverter thyristor. Thus the commutation failure in the case of a rapid increase in $I d$ required by the speed controller can be prevented.

If the control mode is PI action in the speed-control routine, the constant- $\delta$ control using the table is executed, and finally the lead-angle is fed to the gate pulse generators of the inverter. This routine runs every $60^{\circ}$ in the rotor frequency. 


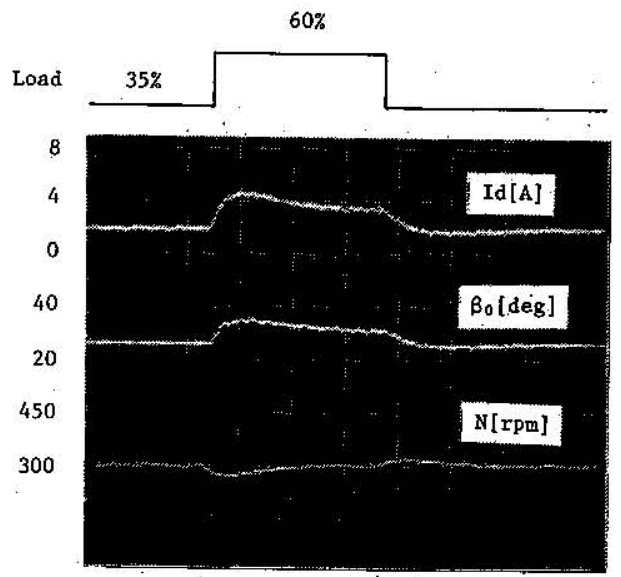

Fig. 14. Responses of de current, lead angle, and speed to step load change. Horizontal; 0.5 s/div.

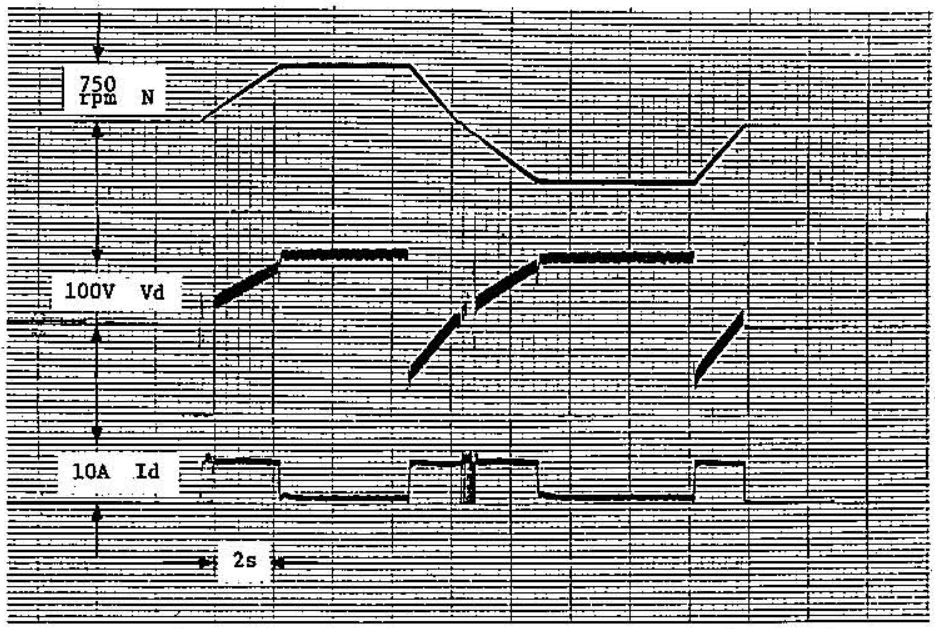

Fig. 15. Waveforms in four-quadrant operation.

\section{EXPERIMENTAL RESULTS}

Fig. 13 shows the responses to a step increase in the speed reference. The lead angle $\left(\beta_{0}-u / 2\right)$ and current $I d$ were maintained at $50^{\circ}$ and $7.5 \mathrm{~A}$, respectively, during the acceleration. Fig. 14 shows the responses to a step load change. Since a step load change could not be detected by the software used, the constant- $\delta$ control was in operation throughout the period. The commutation failure did not occur, although the speed was as slow as $300 \mathrm{r} / \mathrm{min}$. This was mainly due to the fact that the time rate of the rise of the current caused by a step increase in the load was much slower than that caused by a step increase in the speed reference. However, if a heavier step load increase were required, this method might fail. To design gate pulse generators with a minimal delay time and to use them is the best solution for preventing the commutation failure.

Fig. 15 shows the oscillogram in four-quadrant operation. Rapid speed reversal with stable regenerative braking was performed. Fig. 16 shows the characteristics of the torque, and Fig. 17 shows those of the power factor of the ac line input in the two kinds of triggering control schemes, where the curves with a solid line, broken line, etc. represent the theoretical and where $O, \square$, and $\Delta$ represent the tested values. It is obvious

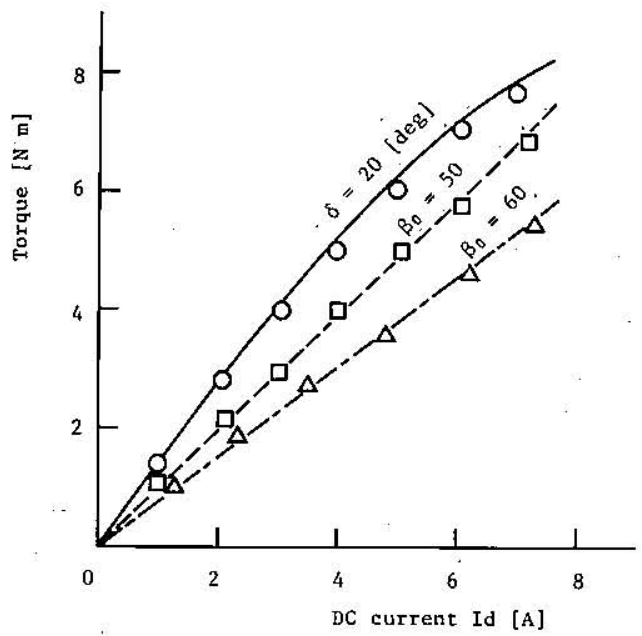

Fig. 16. Torque versus dc current characteristics in constant margin-angle and constant lead-angle schemes.

that the constant- $\delta$ control scheme has an advantage over the constant lead-angle scheme.

\section{CONCLUSION}

The constant margin-angle controlled commutatorless motor and its operating characteristics have been discussed. One 


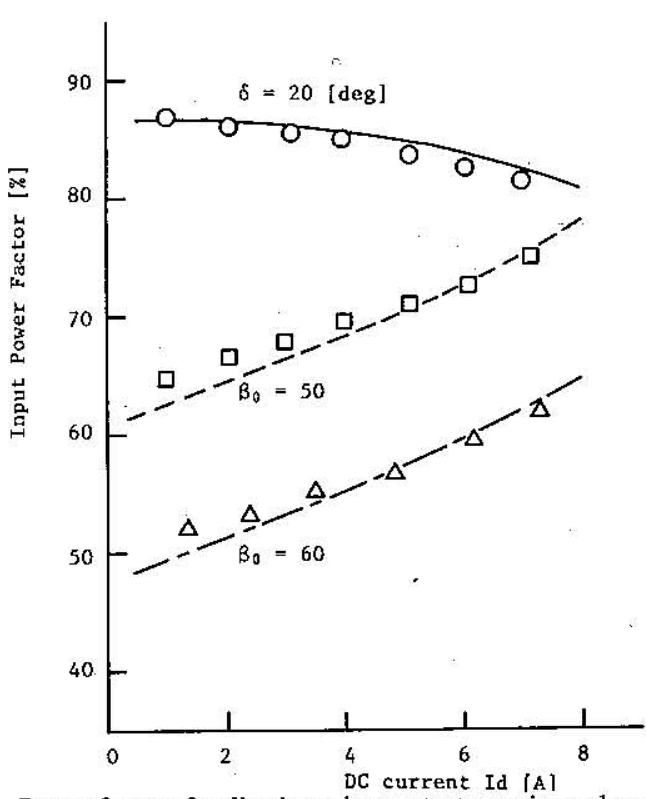

Fig. 17. Power factor of ac line input in constant margin-angle and constant lead-angle schemes.

of the features of the presented scheme is that detection of the margin angle is not needed. Two problems raised by the scheme have been discussed: one is the instability and the other is the variations in the margin angle in a transient state, which is closely related to the commutation capability of the motor. The methods to eliminate the instability and to prevent the commutation failure were presented. Finally, the schemie has been implemented by the use of a microcomputer. Experimental results show that the scheme is efficient and the presented methods are effective for solving the problems.

\section{NOMENCLATURE}

$\beta_{0}$

$\alpha$

$u$

$\delta$

$V d$

$V f$

Ed.

Tav

$T L$

Id

If

$i d d, i d q$

$\omega m$

$R$

$L d c$

$r f$ $r d d, r d q$

Lddo, Ldqo

Laf
Lead angle of commutation defined at noload.

Shift angle of armature voltage due to armature reaction.

Overlap angle of armature currents.

Commutation margin angle $\left(=\beta_{0}-\alpha-\right.$ $u$ ).

DC input voltage.

Field input voltage.

Counter EMF of dc link circuit.

Torque developed.

Load torque.

DC link current.

Field current.

$d$-axis and $q$-axis damper currents, respectively.

Mechanical angular speed of motor.

Resistance of dc link circuit.

Inductance of dc reactor.

Field resistance.

$d$-axis and $q$-axis damper resistances, respectively.

$d$-axis and $q$-axis self inductances, respectively.

Mutual inductance between armature and field windings.
Ladd

$L a d q$

$L f d d$

lf

$L s^{\prime \prime}$

$R \omega$

$J$

$\Psi d o, \Psi q o$

$\Psi d d$

$p$

$P$

[1] S. Fukuda and K. Igarashi, "Control of a commutatorless motor for constant lead angle and constant induced voltage,"' Trans. IEE Japan, vol. 97-B, Oct. 1976.

[2] Y. Takeda, T. Kawakatsu, and T. Hirasa, "Control method combined constant allowable commutation angle with field current on commutatorless motor with induced voltage commutation," Trans. IEE Japan, vol. 98-B, Apr. 1978.

[3] S. Fukuda, Y. Itoh, and A. Nii, "A microprocessor-controlled speed regulator for commutatorless motor drives," Trans. IEE Japan, vol. 104, Sept./Oct. 1984.

[4] H. Le-Huy, A. Jakubowicz, and R. Perret, "A self-controlled synchronous motor drive using terminal voltage system," IEEE Trans. Ind. Appl., vol. IA-18, no. 1, Jan./Feb. 1982.

[5] F. Harashima, K. Iwamoto, and H. Naitoh, "Stability analysis of constant margin-angle controlled commutatorless motor," IEEE Trans. Ind. Appl., vol. IA-19, no. 5, Sept./Oct. 1983.

[6] T. Kataoka and S. Nishikata, "Transient performance of selfcontrolled synchronous motor," IEEE Trans. Ind. Appl., vol. IA-17, no. 2, Mar./Apr. 1981.

[7] S. Fukuda and S. Sasaka, "Stability analysis of constant margin-angle controlled commutatorless motor using lookup table," IEE of Japan Rotating Machines Conf. Record, RM-86-22, July 1986.

[8] S. Fukuda, T. Tenma, and S. Sasaka, "Constant margin-angle control of commutatorless motor using lookup table," IEE of Japan Rotating Machine Conf. Record, RM-86-21, July 1986.

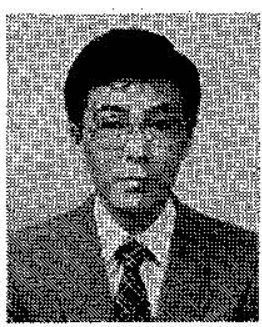

Shoji Fukuda (M'85) was born in Hokkaido, Japan, on May 5, 1942. He received the B.E.E., M.S.E.E., and Ph.D. degrees from Hokkaido University, Sapporo, Japan, in 1965, 1967, and 1977, respectively, all in electrical engineering.

In 1967 he joined the Faculty of Engineering, Hokkaido University, and since 1972 he has been an Associate Professor of the Department of Electrical Engineering, Hokkaido University: During 1981 to 1983, he stayed at University of Saskatchewan, Saskatoon, Canada, as a Postdoctoral Fellow. He has been engaged in research on current source converters/inverters and ac motor drive systemis.

Dr. Fukuda is a member of the Institute of Electrical Engineers of Japan.

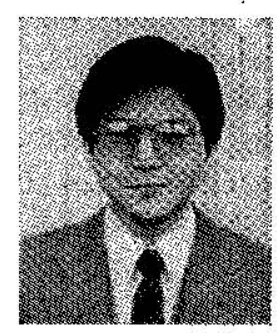

Shinji Sasaka was born in Hokkaido, Japan, on March 25, 1961. He received the B.E.E. and M.S.E.E. degrees from Hokkaido University, Sapporo, Japan, in 1984 and 1986, respectively, all in electrical engineering.

In 1986 he joined Nippon Steel Corporation Muroran Works, Muroran, Japan. He is now in the section of planning factory automation.

Mr. Sasaka is a member of the Institute of Electrical Engineers of Japan. 